\title{
covid-19 Tracking cancer patients during COVID-19 using structured outcomes and keywords
}

\author{
Authors: Rowan Wathes, ${ }^{A}$ Katherine van Ree, ${ }^{\mathrm{B}}$ Gareth Gwynn, ${ }^{C}$ Phawan Hurhangee ${ }^{\mathrm{D}}$ and Catherine Urch ${ }^{\mathrm{E}}$
}

For the duration of COVID-19, cancer pathways will be affected by the significant loss of elective capacity and increased risk of COVID-19-related morbidity and mortality for cancer patients. Imperial College Healthcare NHS Trust (ICHT) has developed a simple, effective MDT recording process, using keywords, to support the tracking of patients who require treatment prioritisation, repeated clinical/MDT reviews and/or need adjustments to their treatment. Following implementation in April, the percentage of MDT outcomes with keywords recorded was $79 \%$ in June and $77 \%$ for the first two weeks of July. Analysis of the 3,680 MDT outcomes with at least one key word recorded showed that $96 \%$ had the 'intention to proceed' recorded. For $59 \%$ patients, the decision was to 'proceed', $5 \%$ patients are being monitored, $3 \%$ patients have been deferred and $29 \%$ were 'closed'. While this process adds time to busy MDTs, we hypothesise that it will support the tracking and safety-netting of thousands of cancer patients whose care has been affected by the pandemic. The process could easily be implemented in other trusts and adapted for other specialties.

KEYWORDS: COVID-19, MDT outcomes, cancer, tracking, recovery

DOI:10.7861/fhj.2020-0110

\section{Problem}

MDT working is the gold standard for cancer patient management in the UK ${ }^{1}$ and many other healthcare systems, and the MDT meeting outcome is a critical part of the patient record. Good data collection, both for the benefit of the individual patient and for the purposes of audit and research, is one of the core principles of effective MDT working. ${ }^{2}$ MDT outcomes are recorded in different

Authors: ${ }^{\mathrm{A}}$ Consultant, The PSC, London, UK; ${ }^{\mathrm{B}}$ deputy director for surgery, cardiovascular and cancer, and consultant radiologist, Imperial College Healthcare NHS Trust, London, UK; Chead of cancer performance and improvement, Imperial College Healthcare NHS Trust, London, UK; ' quality assurance and audits manager, Imperial College Healthcare NHS Trust, London, UK; Edivisional director for surgery, cardiovascular and cancer, and trust cancer lead, Imperial College Healthcare NHS Trust, London, UK ways at different trusts. Imperial College Healthcare NHS Trust (ICHT) uses the Somerset Cancer Record (SCR), a software application developed by the NHS to support the collection of relevant data throughout a patient's cancer journey. The application is used by over 95 organisations in England. ${ }^{3}$

For the duration of the COVID-19 outbreak, cancer pathways will be affected by the significant loss of elective capacity and patients undergoing active cancer treatment will be at increased risk of COVID-19-related morbidity and mortality due to deferred diagnostics and/or treatment, the adjustment of treatment plans in response to COVID-19 risk, and/or a COVID-19 diagnosis while on active cancer treatment. All trusts managing cancer patients will have made significant adjustments to their cancer pathways to respond to the new capacity pressures and to mitigate the risk of harm wherever possible. It is important that any adjustments to the diagnostic/treatment pathway are recorded clearly for each patient so that each member of the clinical team and the patient understands what adjustments were made, and why, and decisions can be audited (for example, in the event of a clinical incident). In time, the outcome data for patients whose pathways were adjusted due to COVID-19 will also be useful for research purposes - and to inform future changes to guidance during this pandemic, or any subsequent pandemics.

This paper outlines a potential solution for recording and tracking adjusted pathways. It also describes how this solution was developed and implemented, and the early results.

\section{Potential solution}

While there is a wealth of guidance on MDT meeting best practice, $1,2,4,5$ this has not yet been updated to reflect the information that needs to be recorded during COVID-19. ICHT has responded by developing a simple and effective MDT recording process to support the tracking, in the Somerset Cancer Register, of:

> patients who need to be prioritised for any available elective capacity

> patients who require repeated clinical/MDT reviews where treatment is not immediately possible

$>$ treatment plans adjusted in response to COVID-19 risk;

> surgical patients eligible for referral to the Cancer Hub Clinical Prioritisation Group (CPG).

MDT leads must ensure that MDT outcomes are structured, including the specified information outlined in Box 1, and contain 
Box 1. Information required for a structured MDT

\section{outcome}

$>$ Imaging (if relevant)

$>$ Histology

> Relevant medical details (eg presentation, co-morbidities)

> Gold standard plan

$>$ COVID-19 plan, including risk assessment

> Outcome keywords (see Fig 1)

(Note: stage and performance status should also be recorded in the relevant fields in Somerset Cancer Record)

keywords to support patient categorisation and report generation (see Fig 1).

The concept of structured outcomes is not new and will be familiar to clinicians. The incorporation of keywords was, however, a response to the need to track patients on adjusted pathways during COVID-19.
The development of the keywords was clinically led, drawing on the wealth of evidence around the quality and safety benefits of comprehensive and accurately coded data, ${ }^{6}$ and involved widespread consultation with ICHT's MDT leads (surgeons and oncologists) and MDT coordinators. The keywords were developed and then trialled in a subset of MDTs. MDT leads and coordinators were asked to add the relevant keywords to the outcome in the 'MDT comments' box on the SCR. They were encouraged to do this during the meeting so that all MDT members could see and comment on what was being recorded. Changes, such as the addition of the 'closed' key word, were incorporated in response to specific feedback. The process was then rolled out to all MDTs through a combination of group introductory sessions with MDT leads and coordinators and one-to-one follow-up sessions. Any outcomes that were not coded during the initial implementation period were retrospectively coded by the Corporate Cancer Team and sent to the MDT leads for checking. Utilisation was tracked and reported on at the MDT leads meeting, a monthly or
1. All patients - intention to proceed

What to record ICHT definition

Proceed The intention is to attempt to actively treat the patient now, subject to elective capacity

Monitor The patient is not suitable for immediate treatment, but will require review during COVID-19

Defer No attempt at active treatment will be made for the duration of COVID-19

Closed No further action required, eg the patient has completed treatment or has a benign diagnosis

2. All patients - treatment intent and likely outcome

\begin{tabular}{|c|c|}
\hline What to record & ICHT definition \\
\hline Emergency & Treatment is required within $24-72$ hours, regardless of likely outcome \\
\hline Curative & A curative outcome is still achievable for the patient at this point in time \\
\hline Adjusted & $\begin{array}{l}\text { The gold standard treatment pathway has had to be adjusted in response } \\
\text { to COVID-19 risk }\end{array}$ \\
\hline Palliative & Palliative intent only \\
\hline
\end{tabular}

3. Surgery patients only - the NHSE surgical priority to support referral to the RMH CPG

What to record NHSE definition

Priority 1a Emergency: operation needed within 24 hours to save life

Priority $1 \mathrm{~b} \quad$ Urgent: operation needed within 72 hours

Priority $2 \quad$ Elective surgery with the expectation of cure. Operation needed within 4 weeks to save life/progression of disease beyond operability, based on: urgency of symptoms, complications (eg local compressive symptoms) biological priority (expected growth rate) of individual cancers.

Local complications may be temporarily controlled, eg with stents, if surgery is deferred and/or interventional radiology

Priority 3 Elective surgery can be delayed for 10-12 weeks with no predicted negative outcome

\section{Example outcomes:}

A patient who is fit for surgery, with a likely curative outcome, who needs treatment within 4 weeks should be categorised as:

Intention to proceed: proceed Treatment intent: curative Surgical prioriy: priority 2

A non-curative patient who is too ill to treat with chemotherapy while COVID-19 infection remains a risk should be categorised as:

Intention to proceed: defer Treatment intent: palliative

A palliative patient undergoing treatment (whose pathway has not been changed as a result of COVID-19) should be categorised as:

Intention to proceed: proceed Treatment intent: palliative

Fig 1. Outcome keywords. Each structured outcome needs to include one key word from section 1 and one from section 2 (and one from section 3 if the patient is having surgery). ICHT = Imperial College Healthcare NHS Trust; NHSE = NHS England; RMP CPG = Royal Marsden Partners Clinical Prioritisation Group. 
bimonthly meeting chaired by the divisional director of surgery, cardiovascular and cancer, where performance and service changes are discussed.

\section{Results}

Use of the keywords increased rapidly: following implementation of the process in April (when no keywords were recorded in this way), the percentage of MDT outcomes with keywords recorded was $79 \%$ in June and $77 \%$ for the first two weeks of July (time of writing). The remainder of management decisions were recorded as outcomes, but without the keywords to support tracking and safety-netting.

Inevitably, there were some MDTs who implemented the process very quickly (for example, prostate, haematology, lung) and others who took longer to adjust. We found that the MDTs who implemented the process quickly had several factors in common: they tended to be those where well-structured outcomes were already being used and there was a strong relationship between the MDT lead and the MDT coordinator.

The main challenge to implementation was scheduling time with the MDT leads and coordinators to explain the process and the anticipated benefits, during a time when staff were busy responding to the pressures caused by COVID-19. Implementation was also complicated by the simultaneous move to virtual MDT meetings and the teething problems associated with that, such as issues with microphone feedback and internet connectivity.

Analysis of the 3,680 MDT outcomes with at least one key word recorded showed that $96 \%$ had the 'intention to proceed' recorded. For $59 \%$ of patients, the decision was to 'proceed', $5 \%$ of patients are being monitored, $3 \%$ patients have had their treatment deferred and 29\% were 'closed' (eg due to a benign diagnosis or completion of treatment). Of the 1,670 with a 'treatment intent and likely outcome' recorded, $5 \%$ patients were recorded as 'emergency', $13 \%$ as 'adjusted', $53 \%$ as 'curative' and $29 \%$ as 'palliative'.

It is important to note that there is clearly a significant number of patients who are yet to enter the MDT system -2 week wait referrals at ICHT are still only at $68 \%$ forecasted levels (weeks commencing 6 and 13 July). This follows a period of referrals dropping to as low as $24 \%$ forecasted levels (week commencing 6 April). The use of keywords does not address this problem, which is being targeted in other ways. For example, the ICHT cancer dashboard now includes referral data by borough (as well as by tumour site). This allows boroughs with lower than expected referral rates to be identified and supported. In addition, the Macmillan GP for Hammersmith and Fulham CCG now attends the MDT leads meeting to share insights from primary care to support discussions around recovery of referral activity.

Initial qualitative feedback from the clinical and management teams has been positive - and a more rigorous assessment of the process is underway. Examples of feedback received so far include:

> 'At this difficult time for both patients and doctors this process was helpful, supportive and informative.' (MDT clinical lead for breast cancer)

> 'The process is really helping us to safety net our patients at a time when there have been significant changes to many of the clinical pathways. It has been easy to roll out and is supported by the clinical teams.' (Senior member of the Corporate Cancer Team)
Reports are now being generated to show which patients have been deferred or are being monitored, and which patients are on an adjusted pathway. These were refined over several weeks to address issues with data entry (relating for example to spelling mistakes and typographical errors). The reports are shared with the MDT leads on a weekly basis so that they can keep track of these patients and restart the patient's cancer pathway when appropriate and when elective capacity allows. Reports can be further refined at tumour level with additional data (eg stage, performance status, demographics) to support the management of a potentially large list of patients waiting for treatment or being monitored for disease progression. The reports are also being used to inform procurement decisions about independent sector capacity.

\section{Conclusions and potential future}

This process has given the clinical teams, and the Corporate Cancer Team, improved oversight of patients under ICHT's care. While it was developed to support the tracking and safety-netting of patients during the pandemic, we expect that the concept of using keywords in MDT outcomes will continue to be useful postCOVID - for example in monitoring compliance with protocolised pathways. The approach could also be extended to other specialties.

The process was straightforward to implement and use of the keywords increased rapidly. This was felt to be down to a combination of careful framing of the process as a way of supporting better safety-netting and oversight of patients during the unprecedented challenges of the pandemic, strong clinical leadership, and close working with business intelligence colleagues on the development and design of the reports.

The process is only useful if clinicians and the Corporate Cancer Team use the reports to actively safety-net patients - for example by ensuring no patient whose management is deferred is 'lost' and all are scheduled for re-review when capacity increases or the risks relating to COVID-19 subside. Further work is needed to fully embed them into ICHT's existing patient management and governance structures.

The process does also add time to already busy MDTs. Department of Health data shows a $20 \%$ year-on-year increase in the number of patient discussions had within an MDT meeting between 2011 and 2014/15. 'The impact of the additional time required to record keywords will need to be monitored as referrals return to normal levels and the number of patients listed for each MDT meeting increases again. However, our hypothesis is that better structuring of MDT outcomes and better coding of decisions will ultimately lead to fewer repeat discussions and more time available for the discussion of each patient. This hypothesis will need to be tested in due course.

ICHT will continue to iterate the approach in the face of subsequent waves of infection. Ideas for future changes include:

> Moving to a drop-down menu data entry approach rather than a free-text approach. This will increase the accuracy of data entry and reporting.

> Iteration of the elements of the structured outcome, potentially to include further information (pending consultation with the MDT leads, coordinators and other stakeholders) such as 'treatment location' (so that it is clear whether the patient is being treated within the Trust or at another trust), "person 
responsible for informing patient about plan', and 'indications for re-discussion (if any)'.

> Further refinement of the reports to ensure they are a useful tool for the MDT leads and coordinators - for example by highlighting patients who were coded as 'proceed' but are yet to have received treatment.

\section{References}

1 Gray R, Gordon B, Meredith M. Meeting patients' needs: improving the effectiveness of multidisciplinary team meetings in cancer services. Cancer Research UK, 2017. www.cancerresearchuk.org/sites/ default/files/full_report_meeting_patients_needs_improving_the_ effectiveness_of_multidisciplinary_team_meetings_.pdf.

2 National Cancer Action Team. The characteristics of an effective multidisciplinary team (MDT). NCAT, 2010. Available from www. ncin.org.uk/view?rid=136
3 Somerset NHS Foundation Trust. Somerset Cancer Register. www. somersetft.nhs.uk/somerset-cancer-register/ [Accessed 25 Sep 2020].

4 NHS England and NHS Improvement. Streamlining multi-disciplinary team meetings: guidance for cancer alliances. NHSE, 2019. www. england.nhs.uk/wp-content/uploads/2020/01/multi-disciplinaryteam-streamlining-guidance.pdf.

5 Royal College of Radiologists. Cancer multidisciplinay team meetings - standards for clinical radiologists. RCR, 2014. www.rcr.ac.uk/ sites/default/files/bfcr1415_mdtms_revised_web_final.pdf.

6 Swinglehurst D, Greenhalgh T. Caring for the patient, caring for the record: an ethnographic study of 'back office' work in upholding quality of care in general practice. BMC HHHealth Serv Res 2015;15:177.

Address for correspondence: Dr Rowan Wathes, The PSC, 30 Stamford Street London, London SE1 9LQ, UK.

Email: rowan.wathes@thepsc.co.uk 